\title{
Health Promotion by Antioxidants
}

\author{
*Hoyoku Nishino ${ }^{1,2}$, Michiaki Murakoshi ${ }^{1,3}$, Yoshiko Satomi ${ }^{4}$
}

${ }^{1}$ KyotoPrefectural University of Medicine, Kawaramachi-Hirokoji, Kamigyo-ku, Kyoto 6028566, Japan; ${ }^{2}$ Ritsumeikan University, Noji-higashi 1-1-1, Kusatsu, Shiga 525-8577, Japan; ${ }^{3}$ Lion Corporation Tajima 100, Odawara, Kanagawa 256-0811, Japan; ${ }^{4}$ SuzukaUniversity of ${ }^{4}$ Medical Science Minamitamagaki-cho 3500-3, Suzuka, Mie 513-8670, Japan

*Corresponding author: Hoyoku Nishino, MD, PhD, Kyoto Prefectural University of Medicine Kawaramachi-Hirokoji, Kamigyo-ku, Kyoto 602-8566, Japan

Submission date: November 14, 2011; Acceptance date: December 15, 2011; Publication date: December, 30, 2011

Running title: Health Promotion by Antioxidants

\section{ABSTRACT:}

Background: Various antioxidnats from daily foods are expected to prevent lifestyle-related diseases. For example, natural carotenoid beta-cryptoxanthin seems to be a promising antioxidant, and based upon epidemiological data it was shown to be a possible cancer preventing agent. For this reason, we chose to study beta-cryptoxanthin more extensively.

Methods and Results: From the result of clinical trial using beta-cryptoxanthin-enriched Mandarin orange juice, it was proven to potentiate the preventive activity of multi-carotenoid mixture against liver cancer in the patients with chronic viral hepatitis-induced liver cirrhosis. Furthermore, beta-cryptoxanthin also has preventive activity against alcohol-induced gammaGTP elevation, and obesity.

Conclusion: An antioxidant beta -cryptoxanthin seems to be valuable for health promotion.

Key words: beta-Cryptoxanthin, Health promotion, Liver cancer prevention, Prevention of alcohol-induced gamma-GTP elevation, Prevention of obesity.

\section{INTRODUCTION:}

Lifestyle-related diseases, such as cancer, metabolic syndrome, alcohol-induced liver dysfunction, and others, are constantly increasing. Therefore, development of the methods to prevent these lifestyle-related diseases is very important. It is our aim to aid in lowering the 
incidence of these diseases through the use of various food factors which include carotenoids, flavonoids, terpenoids, etc. Previously in our laboratory, we found that an antioxidant betacryptoxanthin is a multi-functional food factor, and seems valuable in controlling various lifestyle-related diseases.

Since the incidence of hepatocellular carcinoma in chronic viral hepatitis patients with cirrhosis has been known to be very high, it would be valuable to find an effective method for its prevention. Although interferon treatment has been proven to be effective, many patients are non-responsive and/or intolerable to its strong side effects. Therefore, alternative methods may prove beneficial and should be developed. It has been suggested that diets rich in fruits and vegetables can reduce the risk of various types of cancer, and that some phytochemicals in fruits and vegetables may play important roles for this risk reduction. For instance, carotenoids have been studied as possible candidates for cancer prevention. Initially, carotenoids were thought to be important precursors of vitamin A. In this context, beta-carotene has been studied most extensively, because beta-carotene has the highest provitamin A activity among carotenoids. However, it has been suggested that beta-carotene itself could have a protective effect against cancer without converting to vitamin A [1]. Then, it was shown that various carotenoids other than beta-carotene may also play important role in cancer prevention. Our laboratory has found that alpha-carotene and lycopene showed higher activity than beta-carotene in the suppression of tumorigenesis in various organs [2]. And we have found that a mixture of carotenoids, consisting of lycopene, alpha-carotene and beta-carotene and minor portions of other carotenoids such as phytoene and phytofluene, prevents liver cancer in patients with chronic viral hepatitis and cirrhosis. In addition to alpha-carotene and lycopene, other carotenoids, such as betacryptoxanthin, also seem to be promising carotenoids. Because beta-cryptoxanthin was shown to be a possible candidate as a cancer preventor, based on many epidemiological data [3-9]. Furthermore, besides these carotenoids, various other phytochemicals, including myo-inositol, were also shown to have cancer preventive activities [10]. Based on these data, we examined the effect of the administration of a mixture of beta-cryptoxanthin and myo-inositol in addition to above mentioned carotenoid mixture (lycopene, alpha-carotene and, and beta-carotene) on liver carcinogenesis to improve the efficiency for liver cancer prevention in patients with chronic viral hepatitis and cirrhosis. In this report, brief summary of the study is shown. beta-Cryptoxanthin has also been found to prevent metabolic syndrome, and alcohol induced liver dysfunction. Some of these effects of beta-cryptoxanthin is also shown and discussed.

\section{MATERIALS AND METHODS:}

1. Animal experiment: Spontaneous liver carcinogenesis model was used. Male $\mathrm{C} 3 \mathrm{H} / \mathrm{He}$ mice that have a high incidence of spontaneous liver tumors were treated for 40 weeks with alphaand beta-carotene (at the concentration of $0.05 \%$, mixed as emulsion into drinking water) or vehicle as a control.

2. Clinical study: The study protocol was approved by the review board for research on human subjects, and written informed consent was obtained from each patient. 
In the first trial, patients who have viral hepatitis with cirrhosis were randomly assigned to two groups. Since the study was randomized, the groups were well-balanced with respect to the stage of hepatitis, cirrhosis and other fundamental status. Furthermore subject compliance was assessed at each visit for clinical follow up. Patients in Group 1 were administered the carotenoids mixture, in addition to conventional anti-symptomatic treatment. Patients in Group 2 (the control group) were treated with anti-symptomatic treatment alone. These groups were followed up for $2 \sim 5$ years (3.4 years in average), and hepatocellular carcinoma development was clinically analyzed and recorded utilizing imaging and serum marker studies. Cumulative incidence of hepatocellular carcinoma development was plotted by Kaplan-Meier method, and compared between the two groups.

In the second trial, Mandarin orange juice enriched by beta-cryptoxanthin and myo-inositol was administered with the carotenoids mixture used in the first trial. Follow-up schedule was the same as the first trial.

3. Clinical trial for lifestyle-related diseases using beta-cryptoxanthin-rich Mandarin orange juice: beta-Cryptoxanthin-rich Mandarin orange juice (3mg beta-cryptoxanthin in $80 \mathrm{ml}$ ) was administered to men with obesity (body weight elevation, and high BMI), or with alcohol-induced gamma-GTP elevation as well as obesity.

\section{RESULTS:}

1. Phytochemicals for liver cancer prevention: At first, we evaluated the anti-carcinogenic activity of various natural carotenoids, and found that alpha-carotene showed higher activity than beta-carotene to suppress liver carcinogenesis, as shown in Table 1.

Table 1. Effect of phytochemicals on spontaneous liver carcinogenesis in $\mathrm{C} 3 \mathrm{H} / \mathrm{He}$ male mice

\begin{tabular}{lccc}
\hline Condition & $(\mathrm{n})$ & Mean number of tumors per mouse & (Inhibition \%) \\
\hline Exp. 1 & & 6.3 & \\
Control & $(16)$ & $3.0^{*}$ & $(52 \%)$ \\
+alpha-Carotene & $(17)$ & 4.7 & $(25 \%)$ \\
+ beta-Carotene & $(17)$ & & \\
Exp. 2 & & 7.7 & \\
Control & $(17)$ & $0.9 *$ & \\
+ Lycopene & $(13)$ & & $(88 \%)$ \\
Exp. 3 & $(17)$ & $0.8 *$ & \\
Control & $(13)$ & & \\
+ +myo-Inositol & & & \\
& & & \\
\hline
\end{tabular}

$* \mathrm{p}<0.05$, comparing with control group. 
Exp. 1. alpha-and beta-Carotene: $0.05 \%$ in drinking water, given during the entire experimental period.

Exp.2. Lycopene: $0.005 \%$ in drinking water, given during the entire experimental period.

Exp. 3. myo-Inositol: $1 \%$ in drinking water, given during the entire experimental period.

The mean number of hepatomas was significantly decreased by alpha-carotene treatment as compared with that in the control group. On the other hand, the beta-carotene-treated group did not show a significant difference from the control group, although a tendency toward a decrease was observed.

Furthermore, lycopene was also found to have very high potential for liver cancer prevention (Table 1). In the case of beta-cryptoxanthin, we have found that the plasma level of beta-cryptoxanthin, besides hydrocarbon carotenoids, is lower in liver cancer patients compared with that of healthy individuals, as shown in Table 2.

Table 2. Concentration of beta-cryptoxanthin in serum (Case-control study)

Group (n) Concentration of serum beta-cryptoxanthin $(\mathrm{g} / \mathrm{ml})$

Control (51) $\quad 0.68$

Liver cancer patient

$0.39 *$

${ }^{*} \mathrm{p}<0.05$, comparing with control group.

In addition to carotenoids, other phytochemicals, such as myo-inositol was also found to suppress liver carcinogenesis (Table 1). Studies of cancer prevention by myo-inositol have their origin in the results of inositol hexaphosphate (phytate). It was shown that phytate inhibits carcinogen-induced neoplasia in the colon and mammary gland. Then, it was also found that myo-inositol inhibits tumor formation to a similar magnitude under the same experimental conditions as phytate. And it was pointed out the possibility that dephosphorylated products are actual inhibitors when phytate is given orally, since it was proven that phytate to be quickly absorbed from the stomach and upper small intestine and distributed as inositol monophosphate and myo-inositol. Subsequently, myo-inositol showed very high potential for liver cancer prevention, as shown in Table 1

2. Clinical trial for liver cancer prevention: Based on these pre-clinical results, we have decided to conduct a clinical trial for liver cancer prevention. In the first clinical trial, we evaluated the preventive activity of carotenoid mixture (daily dose of carotenoids: $20 \mathrm{mg}$ in total). Since the preventive potency of lycopene was very high in the experimental study (Table 1), we decided that the majority of the carotenoid in the mixture would be lycopene and thus the 
composition of carotenoids mixture included lycopene $10 \mathrm{mg}$, beta-carotene $6 \mathrm{mg}$, alphacarotene $3 \mathrm{mg}$, and minor portion of carotenoids, such as phytoene and phytofluene, $1 \mathrm{mg}$. These carotenoids were packed with alpha-tocopherol $(50 \mathrm{mg})$ into 4 capsules (Table 3). alphaTocopherol was added to prevent oxidation of carotenoids. However, alpha-tocopherol itself may potentiate the anti-cancer potency of carotenoids mixture.

Table 3. Antioxidant phytochemicals for clinical study

\begin{tabular}{lc}
\hline Phytochemicals & $\begin{array}{c}\text { Dose } \\
\text { (in } 4 \text { capsules) }\end{array}$ \\
\hline Lycopene & $10 \mathrm{mg}$ \\
beta-Carotene & $6 \mathrm{mg}$ \\
alpha-Carotene & $3 \mathrm{mg}$ \\
Other carotenoids & $1 \mathrm{mg}$ \\
alpha-Tocopherol & $50 \mathrm{mg}$ \\
\hline
\end{tabular}

In the 4th annual analysis, significant reduction of hepatocellular carcinoma development was observed in Group 1 (treated group). More than 50\% suppression was found at 4 years of carotenoid intervention; i.e., cumulative incidence of hepatocellular carcinoma in Group 1 was $12.3 \%$, while that in Group 2 (control group) was $34.6 \%$.

Then, as the second clinical trial, we tried to improve the efficacy for liver cancer prevention. It is of interest that mandarin oranges contain possible candidates for liver cancer prevention, such as beta-cryptoxanthin and myo-inositol [9]. We decided to use a formula of juice for the clinical trial, which has been designed to contain $3 \mathrm{mg}$ of beta-cryptoxanthin and $1 \mathrm{~g}$ of myo-inositol in $190 \mathrm{ml}$ canned juice (Table 4).

Table 4. Content of beta-cryptoxanthin and myo-inositol in Mandarin orange juice for clinical trial

\begin{tabular}{lc}
\hline Phytochemicals & $\begin{array}{c}\text { Dose } \\
\text { in one can }(190 \mathrm{ml})\end{array}$ \\
\hline $\begin{array}{l}\text { beta-Cryptoxanthin } \\
\text { myo-Inositol }\end{array}$ & $\begin{array}{l}3 \mathrm{mg} \\
\mathrm{g}\end{array}$ \\
\hline
\end{tabular}

Patients with viral hepatitis and cirrhosis were administered this preparation (one can per day), in addition to a capsule of carotenoid mixture. The patients were followed up at the clinic at regular intervals. Cumulative incidence of hepatocellular carcinoma development was compared with 
that in the group without treatment (Control group). In the data analysis at year 2.5, cumulative incidence of liver cancer in beta-cryptoxanthin and myo-inositol-enriched orange juice with carotenoids mixture capsules-treated group was lower than that in the control group $(\mathrm{p}=0.05)$, as shown in Table 5. The compliance of this intervention study was very good.

Table 5. Liver cancer prevention by beta-cryptoxanthin and myo-inositol-enriched Mandarin orange juice plus carotenoids capsule

\begin{tabular}{|c|c|c|c|}
\hline Group & $(\mathrm{n})$ & $\begin{array}{l}\text { Cumulative incidence } \\
\text { of liver cancer }\end{array}$ & (Inhibition \%) \\
\hline
\end{tabular}

Control group

Group treated with

beta-cryptoxanthin and myo-inositol-

enriched Mandarin orange juice

plus carotenoids capsule

\section{2}

$4.2 *$
$(81 \%)$

Data analysis at 2.5 years of clinical intervention

$*_{\mathrm{p}}=0.05$, comparing with control group.

3. Clinical trial for lifestyle-related diseases prevention using beta-cryptoxanthin-rich Mandarin orange juice: We have also found that $\beta$-cryptoxanthin-rich Mandarin orange juice has preventive activity against alcohol-induced gamma-GTP elevation, and obesity (body weight elevation, and high BMI), and as shown in Table 6 and 7.

Table 6. Effect of beta-cryptoxanthin-rich Mandalin orange juice administration on gamma-GTP level, Body weight, and BMI.(n=7)

\begin{tabular}{lccc}
\hline Condition & Before & 12 Weeks of intervention & $(\mathrm{p}$ value $)$ \\
gamma-GTP & 85.5 & 73.7 & $(\mathrm{p}<0.005)$ \\
Body weight & 74.4 & 72.9 & $(\mathrm{p}<0.001)$ \\
BMI & 27.2 & 26.6 & $(\mathrm{p}<0.001)$ \\
\hline
\end{tabular}

Table 7. Effect of beta-cryptoxanthin-rich Mandalin orange juice administration on body weight, and BMI. $(\mathrm{n}=60)$

\begin{tabular}{lccc}
\hline Condition & Before & 12 Weeks of intervention & $(\mathrm{p}$ value $)$ \\
Body weight & 70.6 & 70.2 & $(\mathrm{p}<0.001)$ \\
BMI & 26.9 & 26.4 & $(\mathrm{p}<0.001)$ \\
\hline
\end{tabular}




\section{DISCUSSION:}

Analysis of action mechanism for multi-functions of beta-cryptoxanthin is very important. As beta-cryptoxanthin is one of the natural antioxidants, protective activity against the excess oxidative stress may play some role. However, further different mechanisms may also important. Thus, a comprehensive analysis using DNA array and protein-antibody array seemed to be valuable. For example, DNA array analysis for beta-cryptoxanthin showed the significant induction of expression in various important genes, such as $R b, p 16$ and $p 73$. It is of interest that the pattern of modulation for DNA and protein expression has been proven to be unique and specific for each antioxidant and phytochemical.These results support the strategy of combination administration of multiple antioxidants and phytochemicals against cancer. In conclusion, combinational use of antioxidants and phytochemicals seems to be a promising method for the prevention of liver cancer in hepatitis virus infected patients with cirrhosis. Further clinical trials are valuable and also needed to confirm and improve the efficacy.

Competing Interests: The authors have no financial interests or conflicts of interest.

Authors's Contributions: All authors contributed to this study equally.

Acknowledgements: This study was carried out in collaboration with research groups of Kyoto Prefectural University of Medicineand other Universitiesand Institutes,including National Cancer Center Research Institute, National Shikoku Cancer Center, Fruit Tree Research Institute, and many Institutes of Companies. This work was supported in part by grants from Council for Advancement of Fruit Tree Science, ProBRAIN, and Ministry of Education, Culture, Sports, Science and Technology, Japan.

\section{REFERENCES:}

1. Peto R et al: Can dietary beta-carotene materially reduce human cancer rates? Nature, 290, 201-208, 1981.

2. Murakoshi M et al: Potent preventive action of alpha-carotene against carcinogenesis: Spontaneous liver carcinogenesis and promoting stage of lung and skin carcinogenesis in mice are suppressed more effectively by alpha-carotene than by beta-carotene. Cancer Res, 52, 6583-6587, 1992.

3. Voorrips LE et al: A prospective cohort study on antioxidant and folate intake and male lung cancer risk. Cancer Epidemiol Biomarkers Prev, 9, 357-365, 2000.

4. Yuan JM et al: Prediagnostic levels of serum beta-cryptoxanthin and retinol predict smokingrelated lung cancer risk in Shanghai, China. Cancer Epidemiol Biomarkers Prev, 10, 767-773, 2001.

5. Yuan JM et al: Dietary cryptoxanthin and reduced risk of lung cancer: The Singapore Chinese Health Study. Cancer Epidemiol Biomarkers Prev, 12, 890-898, 2003.

6. Mannisto $S$ et al: Dietary carotenoids and risk of lung cancer in a pooled analysis of seven cohort studies. Cancer Epidemiol Biomarkers Prev, 13, 40-48, 2004.

7. Goodman MT et al: The assosiation of plasma micronutrients with the risk of cervical atypical 
squamous cells of undetermined significance (ASCUS). Asian Pac J Cancer Prev, 1, 337-345, 2000.

8. Zeegers MP et al: Are retinol, vitamin C, vitamin E, folate and carotenoids intake associated with bladder cancer risk? Results from the Netherlands Cohort Study. Br L Cancer, 85, 977983, 2001.

9. De Stefani E et al: Vegetables, fruits, related dietary antioxidants, and risk of squamous cell carcinoma of the esophagus: A case-control study in Uruguay. Nutr Cancer, 38, 23-29, 2000.

10. Nishino $\mathrm{H}$ et al: Suppression of lung and liver carcinogenesis in mice by oral administration of myo-inositol. Anticancer Res, 19, 3663-3664, 1999. 